\title{
Thermal Effects in Intense Laser-Plasma Interactions
}

\author{
B. A. Shadwick ${ }^{* \dagger}$, G. M. Tarkenton ${ }^{\dagger}$ and E. H. Esarey* \\ ${ }^{*}$ Center for Beam Physics, Ernest Orlando Lawrence Berkeley National Laboratory, University of \\ California, Berkeley, CA 94720 \\ ${ }^{\dagger}$ Institute for Advanced Physics, Suite 199, 10875 US Hwy 285, Conifer, CO 80433
}

\begin{abstract}
We present an overview of a new warm fluid model that incorporates leading-order kinetic corrections to the cold fluid model without making any near-equilibrium assumptions. In the quasi-static limit we obtain analytical expressions for the momentum spread and show excellent agreement with solutions of the full time-dependant equations. It is shown that over a large range of initial plasma temperatures, the fields are relatively insensitive to the pressure force. We discussion implications of this work for model validation.
\end{abstract}

\section{A WARM FLUID MODEL}

We have recently derived a warm fluid model [1] that treats the width of the phasespace distribution asymptotically. This model is particularly appropriate for studying the interaction of a short, intense laser pulse with a cold, collisionless plasma. By exploiting the assumption of small momentum spread, we construct a closed set of moment equations without appealing to a collisional closure. In this model, the phase space distribution is characterized by the moments

$$
\begin{aligned}
n(\mathbf{r}, t) & =\int d^{3} \mathbf{p} f(\mathbf{r}, \mathbf{p}, t), \\
\mathbf{P}(\mathbf{r}, t) & =\frac{1}{n} \int d^{3} \mathbf{p} \mathbf{p} f(\mathbf{r}, \mathbf{p}, t), \\
\Pi_{i j}(\mathbf{r}, t) & =\frac{1}{n} \int d^{3} \mathbf{p}\left(p_{i}-P_{i}\right)\left(p_{j}-P_{j}\right) f(\mathbf{r}, \mathbf{p}, t) .
\end{aligned}
$$

Evolution equations for the moments are derived starting from the Hamiltonian formulation of the Vlasov-Maxwell equations:

$$
\partial_{t} f=\{f, H\}, \quad \partial_{t} \mathbf{E}=\{\mathbf{E}, H\}, \quad \text { and } \quad \partial_{t} \mathbf{B}=\{\mathbf{B}, H\} .
$$

where $H$ is the Hamiltonian

$$
H=m c^{2} \int d^{3} \mathbf{r} d^{3} \mathbf{p} \gamma f+\frac{1}{8 \pi} \int d^{3} \mathbf{r}\left(|\mathbf{E}|^{2}+|\mathbf{B}|^{2}\right),
$$

and $\{\cdot, \cdot\}$ is the non-canonical Poisson bracket. We preform a reduction on the VlasovMaxwell bracket to yield a bracket involving only the moments by assuming that all 
functionals of $f$ can be written as functionals of the moments. Using the chain rule, the bracket is transformed to an expression involving only moments. The Hamiltonian is then approximated using the small momentum-spread assumption and the equations of motion for the moments are obtained from the reduced bracket in the usual way.

The moment bracket is naturally closed and satisfies the Jacobi identity and hence the reduced theory is Hamiltonian. One can show that all Casimirs of the full system map to Casimirs of the moment system. The definition of the moments (1) does not completely determine $f$; many distinct distribution functions can lead to the same values for the moments. By working to lowest order in $\Pi$, the equations of motion are independent of the precise form of the distribution function. Therefore, this theory represents a broad class of possible forms for $f$. In all cases, the Hamiltonian structure of the Vlasov equation is preserved by the moment system. Hence, this theory can be seen as maximally preserving the phase-space structure of the Vlasov system. Moreover, this model is an asymptotic approximation to the Vlasov-Maxwell system with $T / m c^{2}$ as the control parameter. Thus, from this model, we obtain asymptotic approximations to solutions of the full Vlasov-Maxwell equations.

The moments satisfy

$$
\begin{aligned}
\partial_{t} n+\nabla \cdot n \mathbf{u} & =0 \\
\partial_{t} \mathbf{P}+\mathbf{u} \cdot \nabla \mathbf{P} & =q\left(\mathbf{E}+\frac{\mathbf{u}}{c} \times \mathbf{B}\right)-\frac{1}{n} \nabla \cdot \mathrm{p} \\
\partial_{t} \Pi_{i j}+u_{k} \partial_{k} \Pi_{i j} & =-\Pi_{i k} \partial_{j} u_{k}-\Pi_{j k} \partial_{i} u_{k}+\frac{\mathrm{p}_{k i}}{n}\left(\partial_{k} \mathfrak{p}_{j}-\partial_{j} \mathfrak{p}_{k}\right)+\frac{\mathrm{p}_{k j}}{n}\left(\partial_{k} \mathfrak{p}_{i}-\partial_{i} \mathfrak{p}_{k}\right),
\end{aligned}
$$

where $\gamma_{0}=\left(1+P^{2} / m^{2} c^{2}\right)^{1 / 2}, \mathfrak{p}_{i}=P_{i}+q A_{i} / c$ is the canonical momentum,

$$
u_{k}=\frac{P_{k}}{\gamma_{0} m}\left(1-\frac{\Pi_{i i}}{2 \gamma_{0}^{2} m^{2} c^{2}}+\frac{3}{2} \frac{P_{i} \Pi_{i j} P_{j}}{\gamma_{0}^{4} m^{4} c^{4}}\right)-\frac{\Pi_{k i} P_{i}}{\gamma_{0}^{3} m^{3} c^{2}},
$$

and $\mathrm{p}_{i j}=n /\left(\gamma_{0} m\right)\left[\delta_{i k}-P_{i} P_{k} /\left(\gamma_{0}^{2} m^{2} c^{2}\right)\right] \Pi_{k j}$ is the pressure tensor. Since this model is collisionless, the pressure is not forced to be isotropic and, in general, $\mathrm{p}_{i j}$ has significant off-diagonal terms. Note, due to thermal inertia, the fluid advection velocity $\mathbf{u}$ is not simply $\mathbf{P} /\left(\gamma_{0} m\right)$ nor is it, in general, parallel to $\mathbf{P}$. The fields $\mathbf{E}$ and $\mathbf{B}$ are determined Maxwell's equations from the plasma current $\mathbf{j}=q n \mathbf{u}$. The corresponding evolution equations are given by $\partial_{t} \mathbf{E}=\{\mathbf{E}, H\}$ and $\partial_{t} \mathbf{B}=\{\mathbf{B}, H\}$.

\section{WARM QUASI-STATIC RESPONSE}

While in general (4) appear only amenable to numerical solution, if one assumes the plasma response to be quasi-static [2] then considerable analytical progress can be made. We assume that the plasma is underdense $\left(k \gg k_{p}\right)$ and that the laser is non-evolving (i.e., the propagation distance is a fraction of the depletion length). We consider the case of a plasma that is initially in thermal equilibrium with a low temperature (such as a laser-produced channel), i.e., the plasma is initially taken to be isotropic with a temperature on the order of 10 to $20 \mathrm{eV}$ [3-5] and to have negligible bulk motion. Thus 
we have the initial condition $\Pi_{i j}=m T_{0} \delta_{i j}$ implying $\Pi_{i j} /\left(m^{2} c^{2}\right) \sim 10^{-5}$, allowing us to safely neglect the force due to pressure in the momentum equation. Additionally, one can show that in the quasi-static limit, $\mathfrak{p}_{x}=O(\Pi)$, which implies we can ignore the terms involving pressure in the $\Pi$ equations of motion. In this limit, $\Pi$ is driven by the cold, quasi-static fields. Using these approximations and transforming to the comoving coordinates, $(t, z) \mapsto(t, \xi=t-z / c)$, the momentum spread equations become

$$
\begin{aligned}
& \left(1-\beta_{z}\right) \partial_{\xi} \Pi_{x x}=0 \\
& \left(1-\beta_{z}\right) \partial_{\xi} \Pi_{x z}=\Pi_{x x} \partial_{\xi} \beta_{x}+\Pi_{x z} \partial_{\xi} \beta_{z} \\
& \left(1-\beta_{z}\right) \partial_{\xi} \Pi_{z z}=2 \Pi_{z x} \partial_{\xi} \beta_{x}+2 \Pi_{z z} \partial_{\xi} \beta_{z}
\end{aligned}
$$

where $\beta=\mathbf{u} / c$. These equations can be solved analytically to give

$$
\begin{aligned}
& \Pi_{x x}=m T_{0}, \\
& \Pi_{x z}=m T_{0} \frac{\beta_{x}}{1-\beta_{z}}=m T_{0} \frac{n}{n_{0}} \beta_{x}, \\
& \Pi_{z z}=m T_{0} \frac{1+\beta_{x}^{2}}{\left(1-\beta_{z}\right)^{2}}=m T_{0}\left(\frac{n}{n_{0}}\right)^{2}\left(1+\beta_{x}^{2}\right) .
\end{aligned}
$$

To compare our results with the traditional relativistic thermodynamic approach, we force the pressure to be isotropic (as would be the case in collision-dominate fluid). We then have $\mathrm{p}_{i j}=\delta_{i j} n T / \gamma_{0}$ and $\Pi_{i j}=m T\left[\delta_{i j}+P_{i} P_{j} /\left(m^{2} c^{2}\right)\right]$. These assumptions lead to a simple adiabatic equation of state. For $N$ momentum degrees of freedom, $T\left(\gamma_{0} / n\right)^{2 / N}=$ constant, and this implies

$$
\Pi_{i j}=m T_{0}\left(\frac{n}{n_{0} \gamma_{0}}\right)^{2 / N}\left(\delta_{i j}+\frac{P_{i} P_{j}}{m^{2} c^{2}}\right)
$$

where $n_{0}$ and $T_{0}$ are, respectively, the unperturbed density and temperature.

Figure 1 shows (a) the density wave, and (d) the longitudinal electric field driven by a resonant Gaussian laser pulse with frequency $\omega_{0}=20 \omega_{p}$ and dimensionless vector potential $a_{0}=1$ computed using the cold quasi-static model. Fig. 1(b) shows the behavior of $\Pi$ for an initial temperature of $15 \mathrm{eV}$ and (c) shows the corresponding pressure. The behavior of $\Pi_{z z}$ is in qualitative agreement with the thermodynamics of an adiabatic process. This solution exhibits significant anisotropy in the momentum spread and little "heating." Consequently, in this regime, self-trapping of electrons in the wake (leading to dark current) should not be important. Thus, provided the initial plasma temperature is sufficiently small, it should be possible to operate a laser-plasma accelerator without excessive dark current, even at large wake amplitude. Shown in Fig. 1(e) are the components $\Pi$ from (8), i.e., with the assumption that the pressure is isotropic, while Fig. 1(f) shows the isotropic pressure. The clear differences between these results indicate that the assumption of local equilibrium is not justified for this case and, indeed, leads to mis-characterization of phase space. In particular, comparing Fig. 1(c) with Fig. 1(f) 

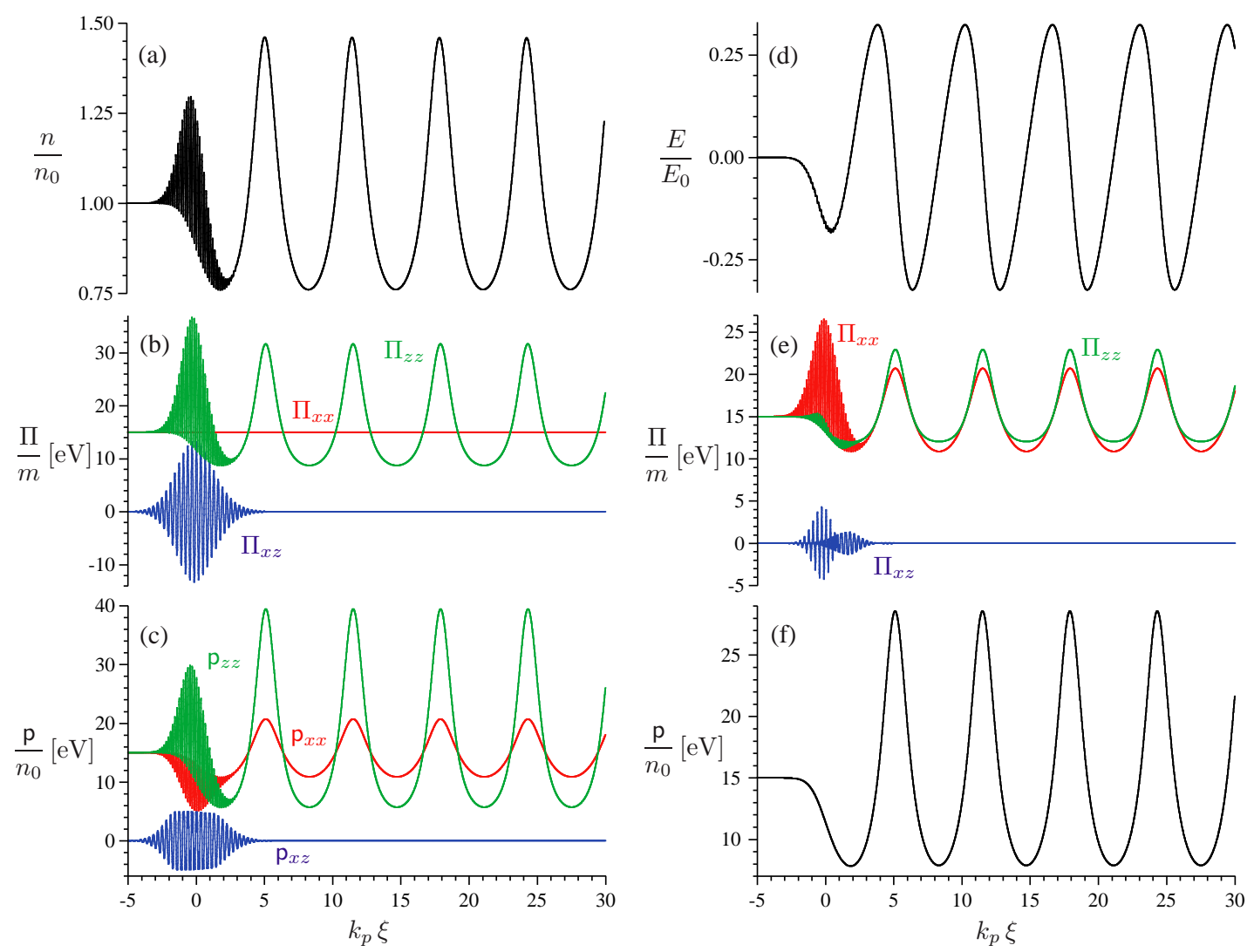

FIGURE 1. Quasi-static plasma response to a resonant Gaussian laser pulse with frequency $\omega_{0}=20 \omega_{p}$ and dimensionless vector potential $a_{0}=1$ : (a) density modulation; (b) $\Pi_{i j}$ from (7); (c) $\mathrm{p}_{i j}$ corresponding to $\Pi$ in (a); (d) longitudinal electric field; (e) $\Pi_{i j}$ from (8), assuming an isotropic pressure; and (f) pressure assuming isotropy.
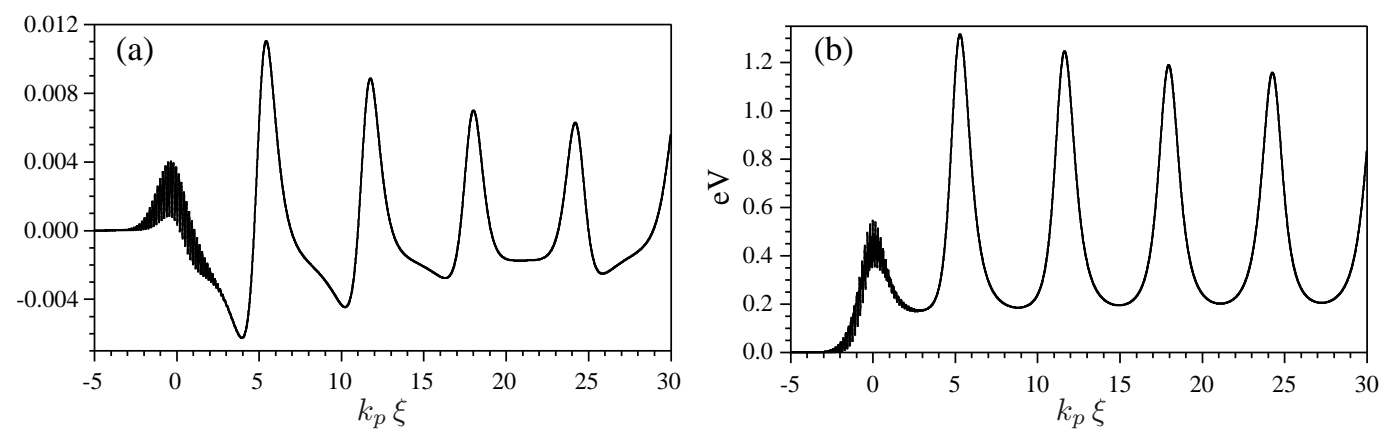

FIGURE 2. Comparison of the quasi-static solutions to numerical solutions of the full time-dependant equations for the parameters of Fig. 1: (a) $\left(n-n^{\mathrm{QS}}\right) / n_{0}$; and (b) $\left(\Pi_{z z}-\Pi_{z z}^{\mathrm{QS}}\right) / m$. The initial plasma temperature was $15 \mathrm{eV}$.

we see that the isotropy assumption overstates the transverse force and understates the longitudinal force.

Figure 2 shows a comparison of the quasi-static results with solutions of the full time-dependent equations. The warm-fluid equations where solved for the parameters of Fig. 1. After the wake was fully-developed, the vector potential, $A_{x}$, was recovered from $B_{y}$ and the quasi-static equations where then solved using $A_{x}$ as the driver. Shown 
in Fig. 2(a) is the difference in quasi-static and time-dependant density while Fig. 2(b) shows the difference in longitudinal momentum spread. Overall we see the agreement is quite good. That the difference in the predictions for the longitudinal momentum spread is much larger than the difference in the densities suggests the quasi-static model somewhat under-predicts heating within the laser.

\section{SHORT PULSE EXAMPLES}

We now turn to solutions of the full time-dependent equations. Figure 3 shows the fields and bulk plasma motion for initial plasma temperatures of $15 \mathrm{eV}, 150 \mathrm{eV}, 1500 \mathrm{eV}$ and $15 \mathrm{keV}$. For this range of initial temperatures, the only noticeable effect on the plasma response is a slight phase shift [most easily seen comparing Fig. 3(a) and Fig. 3(d)]. In the low amplitude case, this phase-shift agrees with the Bohm-Gross [6] dispersion relation modified by the effects of thermal inertial [7]. The corresponding longitudinal momentum spread, $\Pi_{z z}$, is shown in Fig. 4. Except for the overall scale the various $\Pi_{z z}$ curves are nearly identical. The reason for this is clear; even for an initial plasma temperature of $15 \mathrm{keV}$, the pressure force is negligible and the field are essentially unchanged from the those of the cold plasma. Additionally the non-linear terms in $\Pi$ are also negligible, leaving the equation of motion for $\Pi_{i j}$ effectively scaleinvariant with respect to the initial temperature.
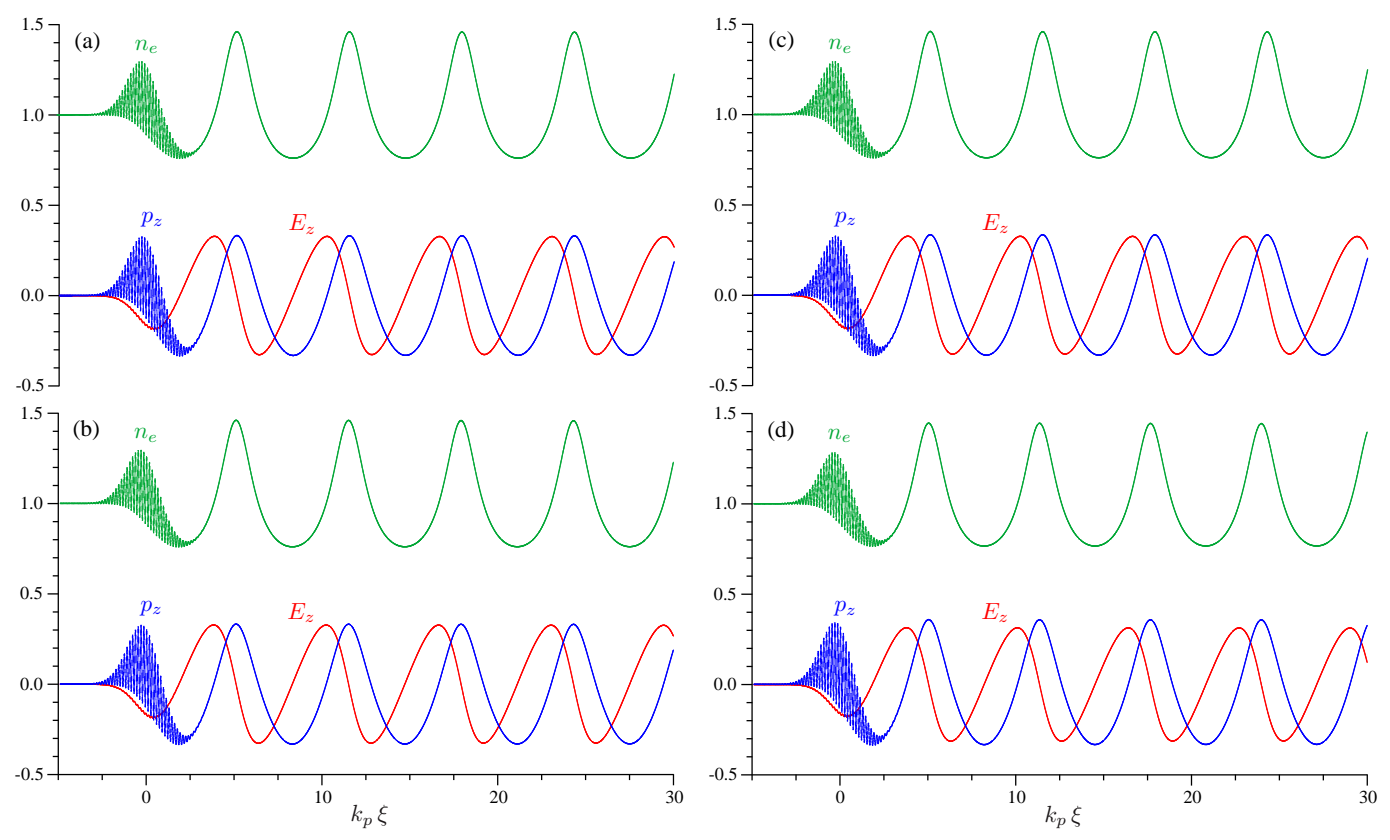

FIGURE 3. Fields and bulk plasma motion obtained by solving (4) numerically for the parameters of Fig. 1. Initial plasma temperature: (a) $15 \mathrm{eV}$; (b) $150 \mathrm{eV}$; (c) $1500 \mathrm{eV}$; and (d) $15 \mathrm{keV}$. Fields shown at $\omega_{p} t=120$. 


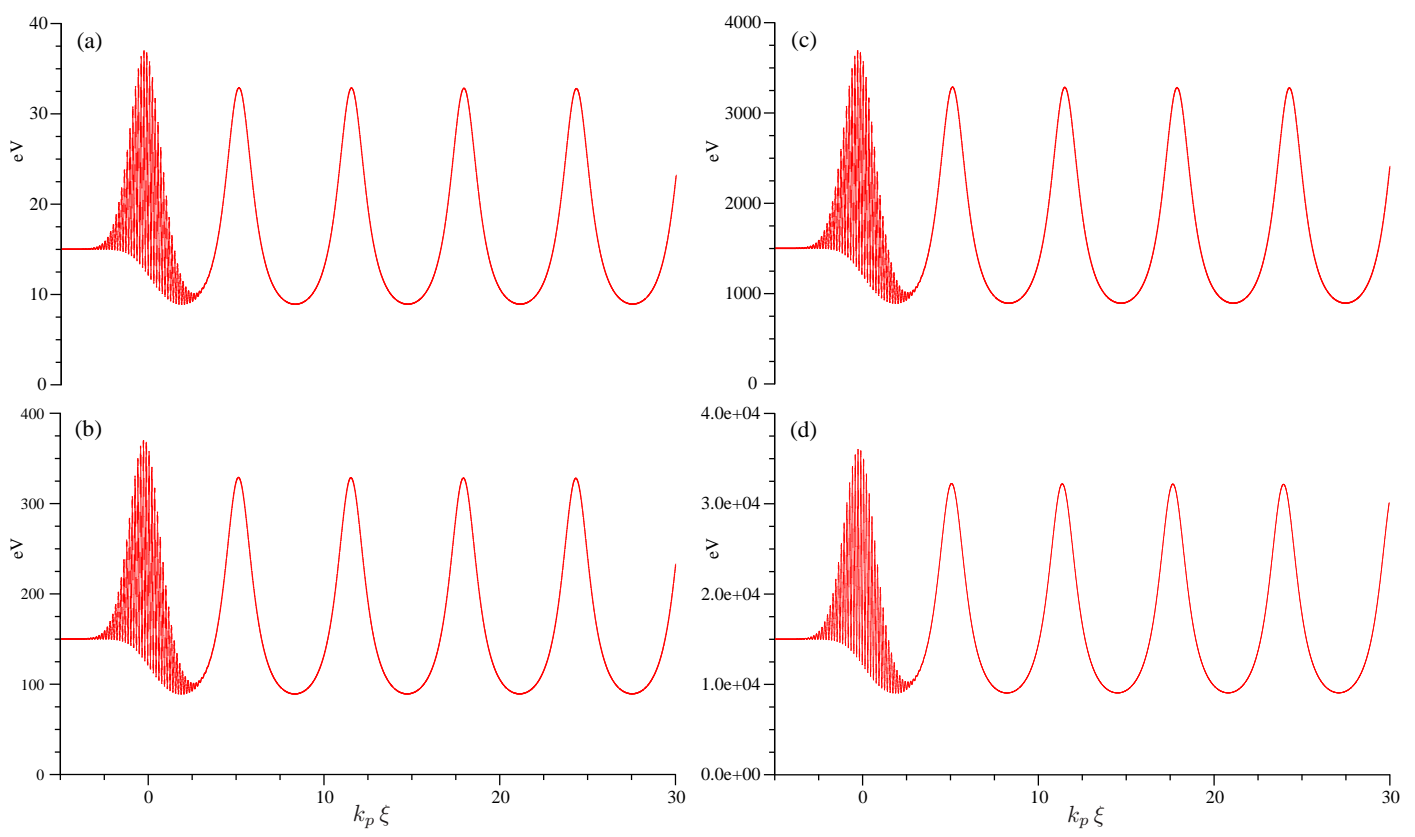

FIGURE 4. Longitudinal momentum spread, $\Pi_{z z} / m$, obtained by solving (4) numerically for the parameters of Fig. 1. Initial plasma temperature: (a) $15 \mathrm{eV}$; (b) $150 \mathrm{eV}$; (c) $1500 \mathrm{eV}$; and (d) $15 \mathrm{keV}$. Results are shown at $\omega_{p} t=120$.

\section{DISCUSSION}

We saw in Fig. 3 that the bulk plasma response, i.e., the density wave and longitudinal electric field, were relatively unchanged over a large range of initial plasma temperatures. This is part of a larger observation that typically is overlooked: The low-order moments of the distribution function and, in particular, the electric and magnetic fields in the Vlasov-Maxwell system are largely insensitive to the details of phase-space. In part this is due to the fields coupling to lowest order moments. In addition, even at large temperatures, the pressure force is small compared to the laser and wake fields. Large-scale aspects of phase space do indeed affect the bulk fields; a trapped particle bunch of high charge will clearly alter the wake field, but the wake will nonetheless be rather insensitive to the particle distribution within the bunch. On the other hand, the microscopic details of phase-space directly affect important phenomena such as particle trapping [8]. In the context of code "Validation and Verification" (a theme of the computational working group at this workshop), the implication of these observations is clear: When attempting to model kinetic effects in a plasma, (via PIC or by other means), it is inappropriate to use the quality of the macroscopic fields as an overall indication of the faithfulness with which phase-space is reproduced. The warm fluid, with its asymptotic relation to the full Vlasov-Maxwell system, provides analytical expressions for the moments of the phase-space distribution in a parameter regime that is of direct interest to the current (and future) generation of laser-plasma experiments. As such it is an excellent "benchmark" for numerical kinetic models such as PIC.

In the case of large mean-free-path, i.e., negligible two-particle correlations, the 
Vlasov equation has a firm theoretical foundation. It enjoys a rigorous derivation from the exact $N$-particle distribution (the Klimontovich distribution [9]) and thus is an exact consequence of the Lorentz force. Consequently, one expects, in the appropriate regime, any discrepancy between experimental results and simulation models to be the result of 1) measurement uncertainty and 2) numerical artifacts in the simulation model. Given this, comparing large scale simulation codes to asymptotic solutions of the Vlasov equation is a necessary step to assess the impact of the latter. The former can be understood by performing sensitivity scans.

\section{CONCLUSIONS}

We have developed a warm, relativistic, fluid theory consistent with representing the kinetic distribution function by second-order moments. This model has a number of interesting features: thermal inertia results in no simple relationship between the average momentum and the advection velocity; the thermodynamics is largely non-relativistic even though the average momentum can be arbitrarily large; and there are no nearequilibrium assumptions. In the quasi-static case we have shown that there is extremely good agreement with the full model.

We have examined the plasma response to a short, intense pulse and found little heating within the laser pulse. There is qualitative agreement with thermodynamic arguments for an adiabatic process: $\Pi_{z z}$ increases where the plasma is compressed and decreases where the plasma is rarified. Moreover, even for very large initial temperature, the density and longitudinal electric field are nearly identical to the cold case.

\section{ACKNOWLEDGMENTS}

The authors gratefully acknowledge numerous discussions with C. B. Schroeder. This work was supported by the Institute for Advanced Physics and by the U. S. DoE under contract No. DE-AC03-76SF0098.

\section{REFERENCES}

1. Shadwick, B. A., Tarkenton, G. M., and Esarey, E. H., Phys. Rev. Lett. (2004), in press.

2. Esarey, E., Sprangle, P., Krall, J., and Ting, A., IEEE Trans. Plasma Sci., 24, $252-288$ (1996).

3. Volfbeyn, P., Esarey, E., and Leemans, W. P., Phys. Plasmas., 6, 2269-2277 (1999).

4. Marconi, M. C., Moreno, C. H., Rocca, J. J., Shlyaptsev, V. N., and Osterheld, A. L., Phys. Rev. E, 62, 7209-7218 (2000).

5. Geddes, C., Esarey, E., Faure, J., Leemans, W., Toth, C., and Tilborg, J. V., Bull. Amer. Phys. Soc., 48, 131 (2003).

6. Bohm, D., and Gross, E. P., Phys. Rev., 75, 1851-1864 (1949).

7. Shadwick, B. A. (2004), in preparation.

8. Schroeder, C. B., Esarey, E., Shadwick, B. A., and Leemans, W. P. (2004), this proceedings.

9. Yu. L. Klimontovich, The Statistical Theory of Non-Equilibrium Processes in a Plasma, MIT Press, Cambridge, MA, 1967. 